Lucie Hüser* und Peter Lehmann

\title{
Resolution enhancement through nearfield-assistance in interference microscopy
}

\author{
Auflösungsverbesserung durch Nahfeld-Unterstützung in der Interferenzmikroskopie
}

DOI 10.1515/teme-2020-0013

Zusammenfassung: Um die Grenzen der optischen Messtechnik zu erweitern und feinere Strukturen messbar zu machen, wurden verschiedene Systeme in der aktuellen Forschung publiziert. Es wurde gezeigt, dass im Nahfeld aufgebrachte Mikrokugeln die Auflösung eines interferometrischen Messsystems verbessern und Strukturen unterhalb Abbe's Beugungsgrenze sichtbar machen können. In dieser Studie werden Messergebnisse mit einem hochauflösenden Linnik-Interferometer unterhalb der Aufösungsgrenze gezeigt. Des Weiteren werden simulative Analysen zum Phasenverhalten im Nahfeld der Mikrokugeln vorgestellt, um die theoretische Erklärung des superauflösenden Verhaltens von Mikrokugeln und die bildgebenden Prozesse des Systems zu ermöglichen.

Schlüsselwörter: Interferometrie, Mikrokugel, SuperAuflösung

Abstract: In order to push the limitations of optical mea-
surement technology further and to make finer structures
measurable, various systems have been published in cur-
rent research. It has been shown that microspheres ap-
plied in the near-field can improve the resolution of an
interferometric measuring system and make structures
below Abbe's diffraction limit visible. In this study, mea-
surement results obtained with a high-resolution Linnik
interferometer show structures below the resolution limit.
Furthermore, a simulative analysis of the phase behavior
in the near-field of the microspheres is presented in order
to find a theoretical explanation of the super-resolution
behavior of microspheres and the imaging processes of the
system.

Keywords: interferometry, microsphere, super-resolution

\footnotetext{
*Korrespondenzautor: Lucie Hüser, Fachgebiet Messtechnik, Fachbereich Elektrotechnik/Informatik, Universität Kassel, Wilhelmshöher Allee 71, 34121 Kassel, E-Mail:

lucie.hueser@uni-kassel.de

Peter Lehmann, Fachgebiet Messtechnik, Fachbereich Elektrotechnik/Informatik, Universität Kassel, Wilhelmshöher Allee 71, 34121 Kassel, E-Mail: p.lehmann@uni-kassel.de
}

\section{Introduction}

Surface metrology with high precision and accuracy is of great importance in the development of metrological processes, both for quality control purposes in industrial aplications and for applications in research. Various methods are used to achieve topographic measurements with a height resolution in the nanometer range. Tactile measurement methods offer high accuracy, but they are not always a preferable method due to long measurement times and possible damage to the measurement object. With optical metrology methods, a resolution in the single-digit nanometer range in the axial direction can be achieved without contact and labeling [4]. In the lateral direction, however, the resolution of a microscope is physically limited by Abbe's diffraction limit, which is given by

$$
d_{\min }=0.5 \frac{\lambda}{\mathrm{NA}} .
$$

with $\lambda$ as the illumination wavelength and NA the numerical aperture of the microscope. The minimum resolvable distance $d_{\text {min }}$ refers to the period length of grating structures, for which Abbe's criterion can be explained physically.

When imaged by a microscope objective, diffraction patterns occur in the Fourier plane, which include the image information. The first order diffraction maxima of a grating include the information of the period length of the structure. The objective lens shows a low-pass filter characteristic with an edge frequency depending on the numerical aperture. Accordingly, structures with highfrequency lateral expansion are no longer imaged by the system.

Our own experimental investigations [10] as well as published studies have shown that a near-field assisting method with the help of microspheres put onto the measurement object is able to improve the resolution of an interference microscope providing information below Abbe's diffraction limit. Wang et al. [27] showed succesful measurements of feature sizes of $50 \mathrm{~nm}$ using a white light microscope with a NA of 0.9 . Other working groups also succeeded in improving the microscopic lateral resolution 
of fine structures using microspheres [3, 25]. Using a white light microscope with a NA of 0.8 Hao et al. [7] resolved a structure of $100 \mathrm{~nm}$ width, whereas Upputuri et al. [2] achieved a lateral resolution of lambda/ 7 .

In interferometric measuring systems it is shown that the super-resolution effect of microspheres can be applied to perform topographic measurements. This was first reported by Wang et al. [26] using a white light interferometer. In $[11,16,17]$ measurement results are presented, which successfully demonstrate the super-resolution by microspheres in interferometric systems of different types. The resulting access to high-frequency image information can be used for profilometry $[18,20]$ and to characterize nanomaterials [13]. Based in there results, further investigations have been made in order to compensate for dispersion in the measuring system [21] or to make the system compact and portable [19].

Photonic Nanojets, which form a very sharp focus on the back side of the microspheres, are frequently used as an explanatory model for the super-resolving capabilities of microspheres $[1,8,28]$. Several studies are published dealing with the engineering of nanojets [22, 23]. Even though many studies use the Photonic Nanojet as an explanatory model for super-resolution, it does not provide a complete theoretical explanation of the imaging processes especially not with regard to high NA measurement instruments $[10,15]$.

This study is intended to investigate the resolution enhancement provided by a microsphere even if the microscope objective lenses of a Linnik interferometer already enables topography measurements with high lateral resolution. Therefore, in the following, measurement results with microspheres as near-field support in a high-resolution Linnik interferometer are presented. The system has a NA of 0.9 and therefore a resolution limit of $255 \mathrm{~nm}$ using a royalblue light source with a central wavelength of $\lambda=460 \mathrm{~nm}$. Experimentally, the smallest structures successfully measured are one-dimensional rectangular grating structures with a period length of $300 \mathrm{~nm}$. The measurement results presented in the following sections demonstrate that microspheres can resolve fine structures with a lateral extension of $230 \mathrm{~nm}$. Furthermore, simulations were carried out in order to investigate the phase transfer behavior of the nearfield and to be able to assess how far these investigations can establish a theoretical explanatory model.

\section{Experimental setup}

The experimental setup used for the measurements presented in this paper essentially consists of a Linnik interferometer with two high-resolution microscope lenses manufactured by Olympus (NA $=0.9,100 \mathrm{x}$ magnification). Figure 1 shows schematic representations of the experimental setup as well as a photograph. For the image acquisition a depth scan in the vertical direction is carried out. As a result, interferograms are obtained during the depth scan for each pixel of the camera, from which the topography of the measurement object is calculated by means of phase or envelope evaluation algorithms [24].
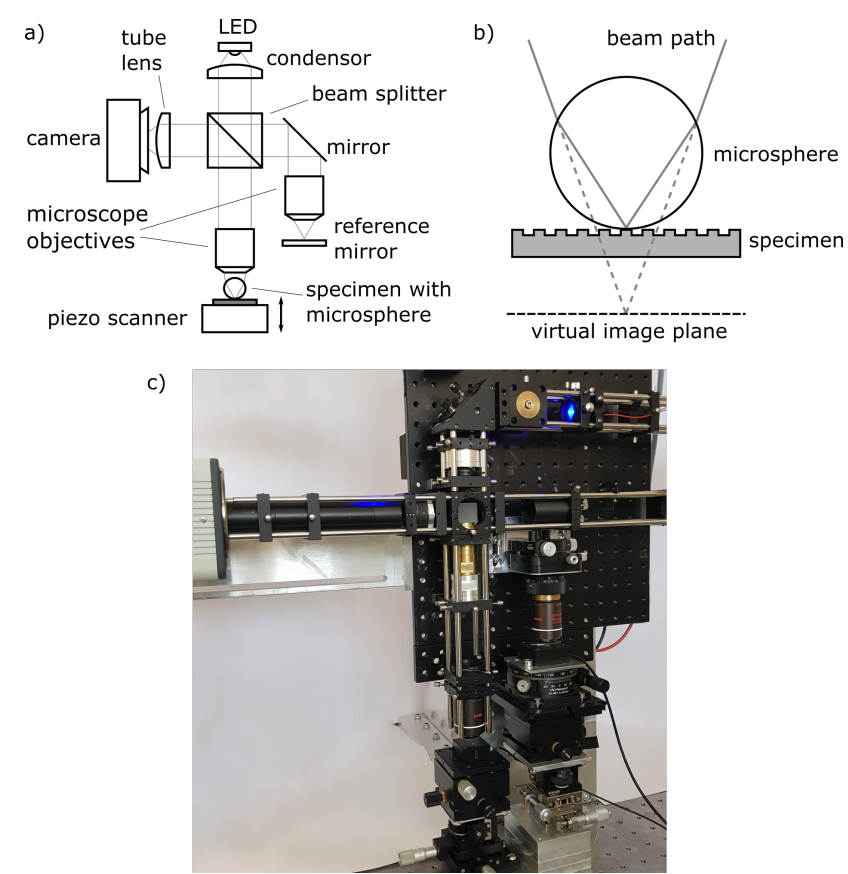

Abb. 1: a) Schematic drawing of the experimental setup; b) Enlarged schematic drawing of the specimen with a microsphere positioned ontop. The beam path through the microsphere creates a virtual image plane; c) Photograph of the experimental setup.

A royal blue LED is used for the measurement results presented here (central wavelength $\lambda=460 \mathrm{~nm}$, LUXEON Rebel Color Line). The camera is a scientific CMOS camera (Hamamatsu, ORCA-flash 2.8) and is used for image acquisition. The depth scan is carried out by a piezo stage manufactured by Physik Instrumente. The reference arm is redirected by a $45^{\circ}$-mirror that results in a vertical optical axis and allows free placement of measurement and reference objects in both arms of the interferometer. Various microspheres were used for the 
near-field support and were positioned on the measurement object. The changed beam path in the near-field, which is illustrated in Figure 1b), creates a virtual image plane located below the image plane without microsphere support. As a result, the topographic structures under the microsphere are measured at different heights in relation to the image plane outside the microsphere.

For the microsphere support, both diameter and material of the microsphere can be varied. So far the best results were obtained using diameters of $5-15 \mu \mathrm{m}$ and $\mathrm{SiO}_{2}$ as material.

\section{Measurement results}

The presented interferometric measuring system is capable of resolving fine grating structures with a period length of $300 \mathrm{~nm}$ without microsphere support. With a microsphere applied in the near-field, structures below Abbe's resolution limit are accessible for topographic measurements.

A suitable measurement object for lateral resolution studies is the Nanoscale Linewidth/Pitch Standard manufactured by Supracon AG [9]. Structures with period lengths of $160 \mathrm{~nm}$ up to $4 \mu \mathrm{m}$ are available in fine gradations on the measurement enabling small-step validation of the super-resolution achieved by the near-field support. For practical reasons, the spheres were placed on the measuring object in an aqueous solution. After the liquid is evaporated, the measurement object with microspheres on it is placed under the interferometer. A depth scan records the interferometric measurement data of the surface and in the virtual image plane. Then topographies are calculated from the measurement data using envelope and phase evaluation [24]. It has been shown that the parameters of the phase evaluation are crucial when evaluating the measurement data $[10,12]$.

In the measurement results presented below, microspheres made of $\mathrm{SiO}_{2}$ with a diameter of $5 \mu \mathrm{m}$ are used. Thus it is possible to successfully measure topographies of one-dimensional grating structures with $230 \mathrm{~nm}$ period length. The results of the phase evaluation are plotted in the topography and the profile section in Figure 2.

Figure $2 \mathrm{~b}$ ) shows the section of the field of view of approximately $2 \mu \mathrm{m}$, which is covered by the microsphere. In the edge area, the high reflections at the surface of the sphere lead to a distortion of the measurement signal and hence to associated phase jumps. In Figure $2 \mathrm{~b}$ ) this can be seen at the right edge of the picture. The profile section shown in Figure 2a) through the central area of the sphere shows the measured height of the structure of $44 \mathrm{~mm}$.
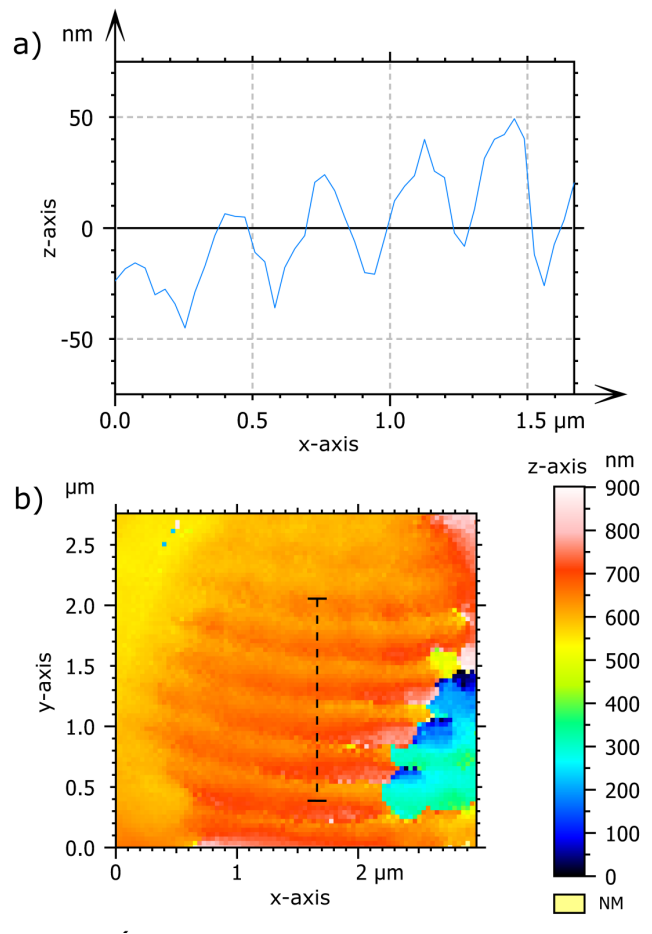

Abb. 2: a) Profile section and b) topography of the phase data ( $650 \mathrm{~nm}$ evaluation wavelength) from the measurement of a $230 \mathrm{~nm}$ structure. Microsphere made of $\mathrm{SiO}_{2}$, diameter $5 \mu \mathrm{m}$.

Note also the additional lateral magnification of 1.5 of the structure, which is associated with the microspheres.

To validate these results, measurements are also carried out with an Atomic Force Microscope (AFM) validated elsewhere $[5,6]$. The results are shown in Figure 3.

The height of the structure in the AFM measurement is $51 \mathrm{~nm}$. In comparison with the microsphere measurements, the height of the structure was measured differently. The lower heigth value in the microsphere measurement can be attributed to the large influence of the evaluation wavelength on the phase evaluation result and the low-pass filtering transfer characteristic of the entire imaging system. In $[10,12]$ investigations are shown which illustrate the direct relationship between the evaluation wavelength and the measured structure height.

\section{Time reversal simulation}

There are already approaches for the theoretical and simulative analysis of the processes involved in the investigation of near-field support to explain the super-resolving ability using microspheres aiming at the Photonic Na- 

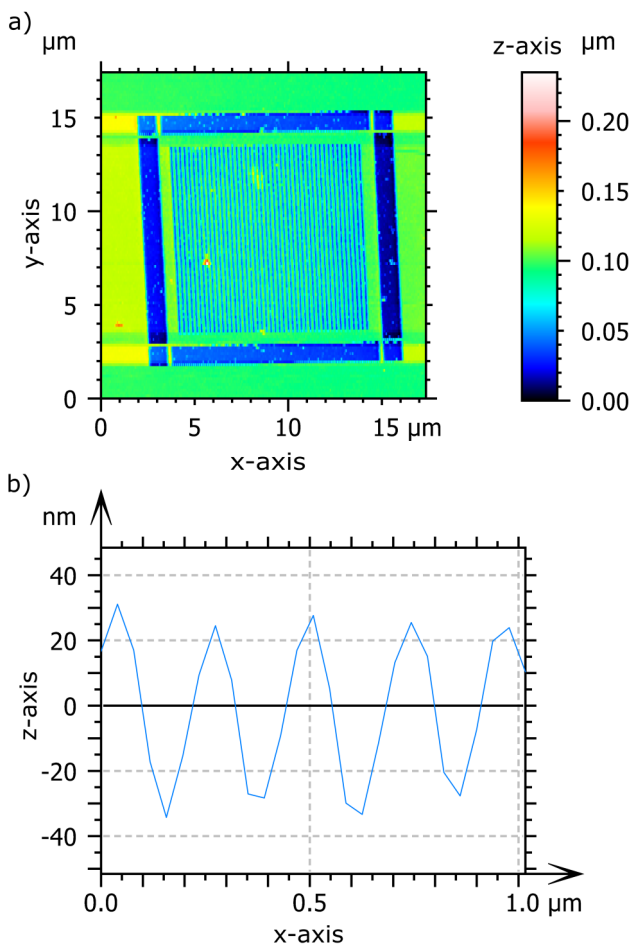

Abb. 3: a) Topography of the $230 \mathrm{~nm}$ grating structure using an AFM; b) Profile section of the measured structure.

nojet $[11,28]$. However, these approaches do not offer a complete simulation of the imaging process and therefore only provide a partial explanation.

The experimental measurement data have shown that high-frequency image information is stored particularly in the phase data. For this reason, the phase behavior of the electromagnetic field distribution in the virtual image plane is investigated in the simulative approach, which is presented below. Not only the illuminating beam path through the microsphere is considered, but also the imaging beam path reflected and diffracted by the measuring object back through the sphere. The simulations are carried out using the Finite Difference Time Domain (FDTD) solver from Lumerical Inc [14].

The simulative setup includes a plane wave illumination and a microsphere on top of a grating structure constituting as the measurement object. As boundary conditions a PML (perfectly matched layer) is used. The field distribution of the scattered field, which includes the imaging beam path back through the sphere, is then recorded and processed further. In order to be able to examine the field distributions and the phase behavior in the virtual image plane in detail, in the second step a time reversal approach is used. The field distribution after the imaging process is used as a new source and the corresponding field distribution in an empty space without a microsphere is calculated. The field and phase data are accessible in the virtual image plane without being limited in the analysis by the different media of microsphere and surrounding material in the near-field of the simulation.

Since in this simulation for the incident plane wave propagates normally, i.e. perpendicular incidence, Abbe's limit leads to $d_{\min }=\lambda / \mathrm{NA}$. Therefore, to be able to investigate the phase behavior close to the resolution limit, a grating with a period length of $600 \mathrm{~nm}$ is chosen. The material of the grating is silicon, the microsphere is made of $\mathrm{SiO}_{2}$ and has a diameter of $10 \mu \mathrm{m}$. The incident plane wave is TM-polarized. For the perpendicular incident case the simulation for a TE-polarized light does not show a significant difference in the phase behavior. Therefore only the results for TM-polarized wave sources are presented.
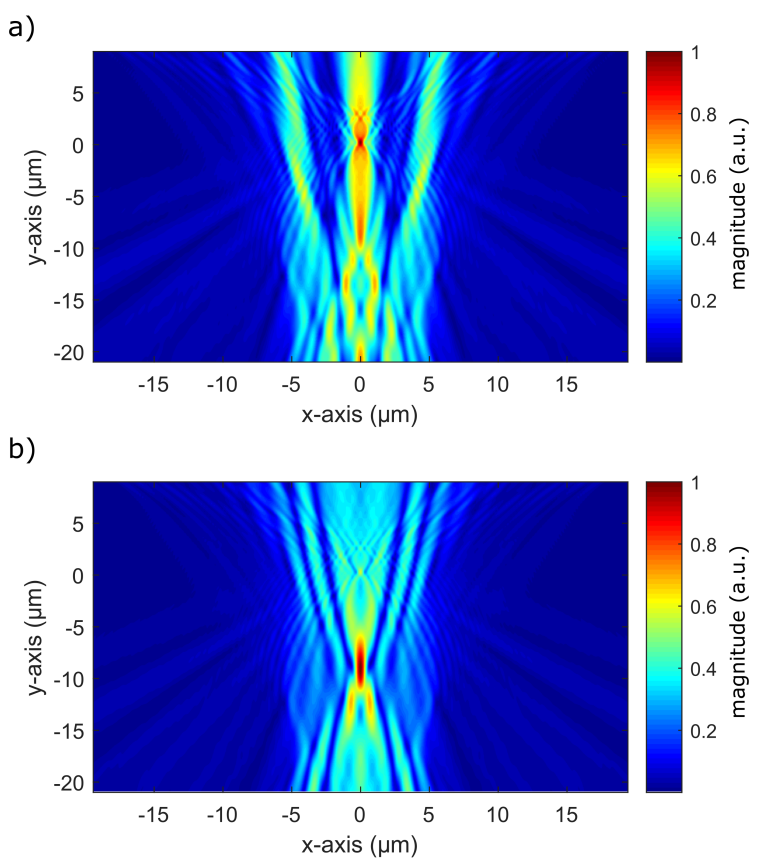

Abb. 4: Magnitude of the electromagnetic field distribution in the time reversal simulation with a microsphere $(d=10 \mu \mathrm{m})$. a) With a grating of $600 \mathrm{~nm}$ period length; b) With a mirror as the measurement object.

The position of the virtual image plane is previously determined by a simulation with a point light source under the microsphere instead of the measurement object. By viewing the intensity distribution in the time reversal simulation, the axial shift of the image plane can be determined. Figure 4 shows the magnitude of the electromagnetic field distributions for the two simulation cases 
regarding a grating (Fig. 4a) and a plane mirror (Fig. 4b) as the measurement object. Figure 5 presents the phase behavior in the virtual image plane. Figure 5a) shows the case including a grating, 5b) without a plane mirror instead of the grating and $5 \mathrm{c}$ ) the difference between the two phase profiles. Considering the phase difference it can be assessed that the image information is contained in the phase unaffected by the phase-changing behavior of the microspheres.

a)

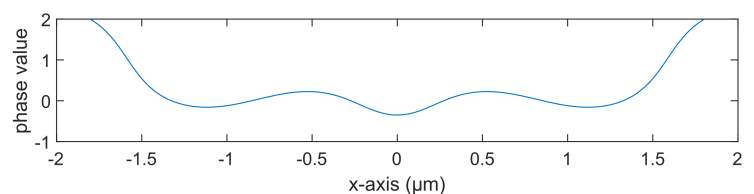

b)

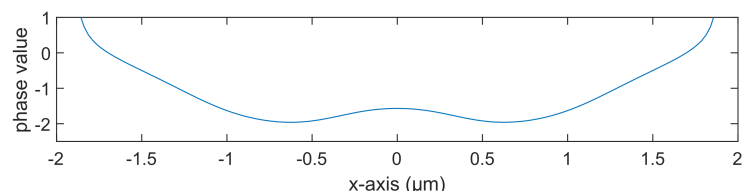

c)

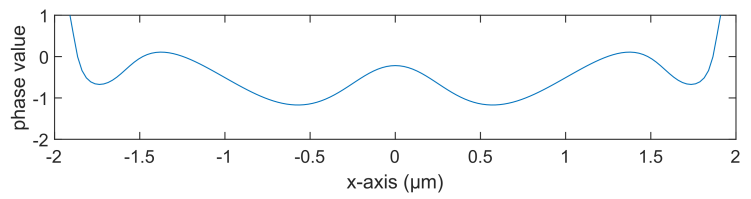

Abb. 5: Analysis of the phase behavior in the virtual image plane a) Microsphere with grating (600 $\mathrm{nm}$ period length); b) Microsphere with mirror; c) Phase difference from a) and b).

It becomes clear that the structure of the grating corresponds to the phase profile in Fig. 5c). An additional magnification of 1.9 can be obtained from the axis scaling of Fig. 5c), which holds for the simulation without a microsphere. This observation provides part of the explanation why the microspheres enable to image structures below Abbe's resolution limit. The simulations underline that the phase data is already manipulated in the near-field. From this it can be concluded that the image information containing the first diffraction orders of the reflection grating is shifted to lower spatial frequencies.

\section{Conclusion}

In this paper, an interferometric measuring system is presented, which is able to measure grating structures with periods below Abbe's diffraction limit using near-field support from microspheres. In particular, the phase evaluation data processing demonstrates that the phase data of the topography measurements contain high-frequency image information of the measured grating structures. Results of a measurement of a grating with a period length of $230 \mathrm{~nm}$ are presented. Additionally, AFM measurements are performed to validate the results on the interferometric measurement.

Furthermore, simulations are carried out in order to examine the phase behavior of the electromagnetic field distribution in the near-field of the sphere. These results show that a manipulation of the phase data, which is crucial for the interferometric measurements, takes place in the near-field. A lateral magnification of the grating structures, which means that high-frequency image data are made accessible to the imaging system such that a lateral resolution slightly below Abbe's resolution limit is achieved. This approach may provide decisive explanations for imaging processes and will be pursued in further studies. In particular, different angles of incidence should be considered especially regarding high NA systems such as a Linnik interferometer as well as the imaging process itself.

Acknowledgement: The presented work was funded by the DFG (German Research Foundation) with the project number LE992/15-1.

\section{References}

[1] Z. Chen, A. Taflove, and V. Backman. Photonic nanojet enhancement of backscattering of light by nanoparticles: a potential novel visible-light ultramicroscopy technique. Optics Express, 12(7):1214, 2004. ISSN 1094-4087. 10.1364/OPEX.12.001214.

[2] A. Darafsheh, G. F. Walsh, L. Dal Negro, and V. N. Astratov. Optical super-resolution by high-index liquid-immersed microspheres. Applied Physics Letters, 101(14):141128, 2012. ISSN 00036951. 10.1063/1.4757600.

[3] A. Darafsheh, N. I. Limberopoulos, J. S. Derov, D. E. Walker, and V. N. Astratov. Advantages of microsphereassisted super-resolution imaging technique over solid immersion lens and confocal microscopies. Applied Physics Letters, 104(6), 2014. ISSN 00036951. 10.1063/1.4864760.

[4] S. Hagemeier and P. Lehmann. Multisensorisches Messsystem zur Untersuchung der Übertragungseigenschaften von Topographiesensoren. tm - Technisches Messen, 85(6):380-394, 2018. ISSN 0171-8096. 10.1515/teme-2017-0138.

[5] S. Hagemeier and P. Lehmann. High resolution topography sensors in a multisenor measuring setup. In SPIE Proceedings, volume 11056, pages 887-895. International Society for Optics and Photonics, SPIE, 2019. 10.1117/12.2525539.

[6] S. Hagemeier, M. Schake, and P. Lehmann. Sensor characterization by comparative measurements using a multi-sensor measuring system. Journal of Sensors and Sensor Systems, 
8(1):111-121, 2019. ISSN 2194878X. 10.5194/jsss-8-1112019.

[7] X. Hao, C. Kuang, X. Liu, H. Zhang, and Y. Li. Microsphere based microscope with optical super-resolution capability. Applied Physics Letters, 99(20):97-100, 2011. ISSN 00036951. 10.1063/1.3662010.

[8] A. Heifertz, S.-C. Kong, A. V. Sahakian, A. Taflove, and V. Backman. Photonic Nanojets. Comput Theor Nanosci., 6 (9):1979-1992, 2009. 10.1166/jctn.2009.1254.Photonic.

[9] U. Huebner, W. Morgenroth, R. Boucher, M. Meyer, W. Mirandé, E. Buhr, G. Ehret, G. Dai, T. Dziomba, R. Hild, and $T$. Fries. A nanoscale linewidth/pitch standard for high-resolution optical microscopy and other microscopic techniques. Measurement Science and Technology, 18(2):422429, 2007. ISSN 13616501. 10.1088/0957-0233/18/2/S14.

[10] L. Hüser and P. Lehmann. Microsphere assisted interferometry with high numerical apertures for 3D topography measurements. Applied Optics, 59(6), 2020. ISSN 1559-128X. 10.1364/ao.379222.

[11] I. Kassamakov, S. Lecler, A. Nolvi, A. Leong-Hoï, P. Montgomery, and E. Hæggström. 3D Super-Resolution Optical Profiling Using Microsphere Enhanced Mirau Interferometry. Scientific Reports, 7(1):1-7, 2017. ISSN 20452322. 10.1038/s41598-017-03830-6.

[12] P. Lehmann, S. Tereschenko, B. Allendorf, S. Hagemeier, and L. Hüser. Spectral composition of low-coherence interferograms at high numerical apertures. JEOS-RP, 15:5(5):1-9, 2019. https://doi.org/10.1186/s41476-019-0101-8.

[13] A. Leong-Hoi, C. Hairaye, S. Perrin, S. Lecler, P. Pfeiffer, and P. Montgomery. High Resolution MicrosphereAssisted Interference Microscopy for 3D Characterization of Nanomaterials. Physica Status Solidi (A) Applications and Materials Science, 215(6):1-7, 2018. ISSN 18626319. $10.1002 /$ pssa. 201700858.

[14] Lumerical. Lumerical inc. URL https://www.lumerical.com/. visited 26.06.2020.

[15] A. Maslov and V. Astratov. Resolution and Reciprocity in Microspherical Nanoscopy: Point-Spread Function Versus Photonic Nanojets. Physical Review Applied, 11(6):064004, 2019. ISSN 2331-7019. 10.1103/PhysRevApplied.11.064004.

[16] P. Montgomery, S. Lecler, A. Leong-Hoi, and P. Pfeiffer. 3D nano surface profilometry by combining the photonic nanojet with interferometry. Journal of Physics: Conf. Series, 794(1): 012006, 2017. 10.1088/1742-6596/794/1/012006.

[17] P. C. Montgomery, S. Lecler, A. Leong-hoï, S. Perrin, and P. Pfeiffer. Sub-diffraction surface topography measurement using a microsphere- assisted Linnik interferometer. Proceedings of SPIE - The International Society for Optical Engineering, 10329(33):1-10, 2017. 10.1117/12.2270223.

[18] P. C. Montgomery, S. Lecler, A. Leong-Hoï, and S. Perrin. High Resolution Surface Metrology Using MicrosphereAssisted Interference Microscopy. Physica Status Solidi (a), 1800761:1800761, 2019. ISSN 18626300. 10.1002/pssa.201800761.

[19] T. O'Connor, A. Anand, and B. Javidi. Field-portable microsphere-assisted high resolution digital holographic microscopy in compact and 3D-printed Mach-Zehnder Interferometer. OSA Continuum, 3(4):1013, 2020. ISSN 2578-7519. 10.1364/osac.389832.
[20] S. Perrin, A. Leong-Hoï, S. Lecler, P. Pfeiffer, I. Kassamakov, A. Nolvi, E. Hæggström, and P. Montgomery. Microsphere-assisted phase-shifting profilometry. Applied Optics, 56(25):7249-7255, 2017.

[21] S. Perrin, Y. J. Donie, P. Montgomery, G. Gomard, and S. Lecler. Compensated Microsphere-Assisted Interference Microscopy. Physical Review Applied, 13(1):1, 2020. ISSN 2331-7019. 10.1103/physrevapplied.13.014068.

[22] C. Rockstuhl, H. P. Herzig, S. Mühlig, M.-S. Kim, and T. Scharf. Photonic nanojet engineering: focal point shaping with scattering phenomena of dielectric microspheres. Integrated Optics: Devices, Materials, and Technologies XV, 7941:794115, 2011. 10.1117/12.887604.

[23] C. Rockstuhl, M.-S. Kim, H. P. Herzig, T. Scharf, and S. Mühlig. Engineering photonic nanojets. Optics Express, 19 (11):10206, 2011. 10.1364/oe.19.010206.

[24] S. Tereschenko. Digitale Analyse periodischer und transienter Messsignale anhand von Beispielen aus der optischen Präzisionsmesstechnik. PhD thesis, Universität Kassel, 2018.

[25] P. K. Upputuri and M. Pramanik. Microsphere-aided optical microscopy and its applications for super-resolution imaging. Optics Communications, 404(March):32-41, 2017. ISSN 00304018. 10.1016/j.optcom.2017.05.049.

[26] F. Wang, L. Liu, P. Yu, Z. Liu, H. Yu, Y. Wang, and W. J. $\mathrm{Li}$. Three-dimensional super-resolution morphology by nearfield assisted white-light interferometry. Scientific Reports, 6 (April):24703, 2016. ISSN 20452322. 10.1038/srep24703.

[27] Z. Wang, W. Guo, L. Li, B. Luk'Yanchuk, A. Khan, Z. Liu, Z. Chen, and M. Hong. Optical virtual imaging at $50 \mathrm{~nm}$ lateral resolution with a white-light nanoscope. Nature Communications, 2(1):216-218, 2011. ISSN 20411723. $10.1038 /$ ncomms 1211 .

[28] H. Yang, R. Trouillon, G. Huszka, and M. A. Gijs. SuperResolution Imaging of a Dielectric Microsphere is Governed by the Waist of its Photonic Nanojet. Nano Letters, 16(8):4862-4870, 2016. ISSN 15306992. $10.1021 /$ acs.nanolett. 6 b01255. 